\title{
A novel tool for visualization and detection of pancreatic neuroendocrine tumours. A 'fluorescent' world is calling for exploration?
}

\author{
Maria Sotiropoulouㄹ, Francesk Mulita², Georgios-loannis Verras², Dimitrios Schizas ${ }^{1}$, \\ Alexandros Papalampros ${ }^{1}$, Levan Tchabashvili², Charalampos Kaplanis², Elias Liolis ${ }^{3}$, loannis Perdikaris², \\ Ioannis Maroulis², Michail Vailas ${ }^{2}$ \\ ${ }^{1}$ Department of Surgery, National and Kapodistrian University of Athens, Athens, Greece \\ ${ }^{2}$ Department of Surgery, General University Hospital of Patras, Greece \\ ${ }^{3}$ Department of Internal Medicine, Division of Oncology, General University Hospital of Patras, Greece
}

\begin{abstract}
Aim of the study: Pancreatic neuroendocrine tumours (pNETs) are rare tumours with a propensity to metastasize. Physicians frequently face a huge clinical challenge during the localization of these lesions. The aim of this study is to investigate whether fluorescence-guided localization techniques with indocyanine green (ICG) can be utilized as a detection tool in pNETs, along with any other clinical implications of this technique.

Material and methods: A thorough literature search in PubMed and Google Scholar, under the terms 'ICG OR Indocyanine OR Fluorescence AND Neuroendocrine' until 31 June 2021, regarding the utilization of indocyanine-fluorescence in localization of pancreatic neuroendocrine, was conducted by the authors, and the associated results are presented.

Results: Indocyanine fluorescence imaging may facilitate the efforts of surgeons to identify occult pancreatic neuroendocrine lesions, assisting them in the identification of resection margins and delineation of the surgical anatomy when it is difficult to clarify.

Conclusions: Indocyanine-fluorescence imaging might play a pivotal role in pancreatic surgery in terms of localization for neuroendocrine tumours. However, further large-scale clinical studies are needed to assess the absolute indications and optimal use of this technique.
\end{abstract}

Key word: cancer, fluorescence, ICG, indocyanine, neuroendocrine, pancreas.

\section{Introduction}

Pancreatic neuroendocrine tumours (pNETs) are a distinct group of neoplasms arising from cells in the diffuse neuroendocrine system, located in the cells of Langerhans. Its incidence reaches 0.5 per 100,000 population [1]. This type of neoplasm might cause the presentation of overt clinical syndromes, based on the type of the hormone they secrete. Active peptides like insulin, gastrin, glucagon, somatostatin, and VIP produced by pNETs are responsible for the development of clinical symptoms, which is the case in functional tumours like insulinoma, gastrinoma, glucagonoma, somatostatinoma, and VIPoma [2]. Interestingly, most of these neoplasms (70\%) do not produce distinct clinical syndromes, secreting minimal amounts of active peptides or no hormones at all $[3,4]$.

Because of the small size and indolent nature of pNETs, physicians and surgeons frequently face a huge clinical challenge during localization work-up either preoperatively or even intra-operatively [5]. Therefore, after biochemical confirmation of their presence, a vari- ety of imaging techniques is nowadays utilized in order to successfully confirm their exact location within the pancreatic gland [6]. Computed tomography is the most commonly used modality, with a mean sensitivity for pNETs of $82 \%$, while magnetic resonance imaging (MRI) has a similar mean sensitivity of $79 \%$, being at the same time more sensitive for the detection of liver metastases [7, 8]. Finally, endoscopic ultrasound EUS and fine-needle aspiration is a useful extra imaging tool that is currently considered the most sensitive test for localizing small pNETs based on recent scientific data [9].

Recently, Indium-111 somatostatin receptor scintigraphy and gallium-68 positron emission tomography, both of which use radiolabelled somatostatin analogues to localize pNETs have brought a revolution in the localization process of pNETs, with the latter becoming the functional imaging modality of choice with sensitivity and specificity for the diagnosis to be $93 \%$ and $91 \%$, respectively [10]. In spite of that, when these imaging modalities are used, pre-operative and intraoperative detection rates are successful in $92 \%$ of cases, whereas localization of tumours less than $2 \mathrm{~cm}$ remains difficult even during surgery [5, 11]. 
Intraoperative ultrasound seems to be beneficial in such cases, and recently fluorescence-guided (FG) surgery has emerged as a novel intraoperative modality to assist surgeons to localize such tumours, sentinel lymph nodes, and adjacent vital structures in real time [12]. The aim of this study is to investigate whether FG localization techniques with indocyanine green (ICG) can be utilized as a detection tool in cases of pNETs, especially when conventional imaging studies fail, along with any other clinical implications of this technique.

\section{General principles of indocyanine green-fluorescence imaging}

A growing body of scientific data have reported that near-infrared (NIR) fluorescence imaging is a promising technique able to facilitate intraoperative, real-time, visual information in many different surgical operations $[13,14]$. Contrast agents that target tumour-specific characteristics are considered prerequisite in order to detect tumours using NIR fluorescence $[15,16]$. Despite that, these novel tumour-specific agents are under development and are not clinically available yet. The only agent currently in use approved by the US Food and Drug Administration is ICG [17]. Although this is not tumour specific, the enhanced permeability and retention (EPR) effect of this agent can be used to obtain accumulation in tumours [18]. Newly formed, more porous blood vessels in cases of malignant tissue growth allow ICG to accumulate in the surrounding tissues. Concurrently, poorly developed lymphatics in the tumour area result in an increased retention of this agent [16].

Fluorescence imaging using ICG as a fluorophore has been applied in cases when it is necessary to assess coronary artery bypass graft patency, visualize cancerous lymph nodes, and identify sentinel lymph nodes during breast and gastric cancer surgeries [19]. Indocyanine green enters hepatocytes and is excreted into bile. Therefore, the technique was initially utilized in hepatobiliary surgery, able to visualize malignant liver lesions, bile ducts during cholecystectomy, and hepatic arterial/portal blood flow [20, 21]. Two separate studies have demonstrated the efficacy in visualization of pancreatic lesions, including $13 \%$ of pancreatic adenocarcinomas and $100 \%$ of pNETs [16, 22].

As far as the basic principles of ICG administration are concerned, ICG is usually administered intravenously before the beginning of the surgical procedure and in various time intervals intraoperatively. This dye becomes fluorescent when excited by light with specific wavelength in the near-infrared radiation (NIR) spectrum. A variety of fluorescence imaging systems exist, able to overlay fluorescence imaging on background colour images by visualizing visible and near-infrared rays $[23,24]$. For open surgery the camera imaging head is usually manually positioned approximately $30 \mathrm{~cm}$ above the pancreas, and interpretation of the exact tumour location and the appropriate resection margins is feasible after intraoperative administration of ICG in non-toxic doses [19].

\section{Usefulness of indocyanine green \\ fluorescence imaging for real-time visualization of pancreatic neuroendocrine tumours}

Scientific data have demonstrated that visualization of the liver perfusion status using ICG fluorescence imaging is feasible, highlighting the fact that liver regions without hepatic artery or portal vein flow do not demonstrate fluorescence $[25,26]$. More specifically, Kawaguchi et al. [26] studied 21 consecutive patients who underwent liver resection with excision of major hepatic veins and were evaluated using ICG fluorescence imaging after clamping veins and injecting ICG. They concluded that ICG fluorescence imaging with hepatic vein clamping visualized non-veno-occlusive, venoocclusive, and ischaemic regions, emphasizing the role of this technique in guiding liver transection by intraoperative navigation. The same team of authors hypothesized that hypervascular lesions such as pNETs can exhibit strong fluorescence images because their vascularity is richer than that of the surrounding parenchyma [19].

To evaluate and support their hypothesis Shirata et al. [19] tried to assess and compared the fluorescence intensity $(\mathrm{FI})$ of pancreatic lesions and the surrounding parenchyma in a group of 23 patients based on the types and vascularity of pancreatic. A fluorescence imaging system (PINPOINT; Stryker, San Jose, CA) was used for open and laparoscopic surgery, while $1 \mathrm{ml}$ of ICG $(2.5 \mathrm{mg}$ ) was administered intravenously, placing a $30^{\circ}$ near-infrared camera $30 \mathrm{~cm}$ above the surgical field. Five patients underwent distal pancreatic resection for neuroendocrine hypervascular tumours that ranged in size between 9.0 and $45.1 \mathrm{~mm}$. They found that the $\mathrm{FI}$ values were significantly higher in pNETs compared with those in the surrounding pancreatic parenchyma, determining the exact location of resection line in order to obtain negative margins. Given the fact that the vascularity of pNETs is richer than that of the surrounding pancreatic parenchyma, pNETs exhibited 1.99 times higher $\mathrm{Fl}$ than the surrounding tissues, making them identifiable in $100 \%$ of cases.

Paiella et al. [22] aimed to explore the results of the application of NIR in the laparoscopic resection of pNETs in a group of 10 patients with ages ranging from 39 to 66 years, who underwent laparoscopic pancreatic surgery (PS) for pNETs. The near-infrared system used in this study was the D-Light P (Karl Storz GmbH e Co. KG, Tuttlingen, Germany), and patients received ICG $25 \mathrm{mg}$ given in 5 boluses of $5 \mathrm{mg}$ each. Indocyanine green-fluorescence imaging identified all 10 pNETs. Nine laparoscopic 
Table 1. The most relevant studies for ICG fluorescence in pancreatic neuroendocrine tumours

\begin{tabular}{|c|c|c|c|c|c|c|c|}
\hline Authors & Patients & Gender & Age & Histology & $\begin{array}{c}\text { Fluorescence } \\
\text { or defect } \\
\end{array}$ & $\begin{array}{c}\text { Indocyanine green - } \\
\text { dose detection }\end{array}$ & $\begin{array}{c}\text { Latency time } \\
\text { (sec) }\end{array}$ \\
\hline \multirow{5}{*}{$\begin{array}{l}\text { Shirata } \\
\text { et al. [19] }\end{array}$} & 1 & $\mathrm{~F}$ & 77 & NET & Fluorescence & $N / R$ & $N / R$ \\
\hline & 2 & M & 47 & NET & Fluorescence & $N / R$ & $N / R$ \\
\hline & 3 & M & 70 & NET & Fluorescence & $N / R$ & $N / R$ \\
\hline & 4 & M & 39 & NET & Fluorescence & $N / R$ & $N / R$ \\
\hline & 5 & $\mathrm{~F}$ & 34 & NET & Fluorescence & $N / R$ & $N / R$ \\
\hline \multirow{10}{*}{$\begin{array}{l}\text { Paiella } \\
\text { et al. [22] }\end{array}$} & 1 & M & 44 & NF-pNET & Fluorescence & 2 & 70 \\
\hline & 2 & M & 66 & NF-pNET & Fluorescence & 3 & 130 \\
\hline & 3 & M & 59 & NF-pNET & Fluorescence & 2 & 75 \\
\hline & 4 & $\mathrm{~F}$ & 45 & Insulinoma & Fluorescence & 2 & 70 \\
\hline & 5 & $\mathrm{~F}$ & 43 & NF-pNET & Fluorescence & 2 & 70 \\
\hline & 6 & $M$ & 41 & Insulinoma & Fluorescence & 2 & 80 \\
\hline & 7 & $\mathrm{~F}$ & 39 & NF-pNET & Fluorescence & 2 & 80 \\
\hline & 8 & $\mathrm{~F}$ & 53 & NF-pNET & Fluorescence & 2 & 70 \\
\hline & 9 & M & 43 & NF-pNET & Fluorescence & 2 & 75 \\
\hline & 10 & $\mathrm{~F}$ & 59 & NF-pNET & Fluorescence & 2 & 70 \\
\hline
\end{tabular}

NET - neuroendocrine tumour, NF-pNET - non-functional pancreatic neuroendocrine tumour, N/R - not reported

distal pancreatectomies with splenectomy and 1 laparoscopic enucleation were performed. Eight non-functioning pNETs and 2 insulinomas were found in the final histological specimen. Ninety per cent of the lesions were detected after the second ICG bolus with a mean latency time of $80 \mathrm{sec}$, while the peak of tumour fluorescence was reached $20 \mathrm{~min}$ after the last bolus. In the case of enucleation, a fluorescent signal was identified at the bed of the initial resection, and therefore a further resection was performed in order to achieve negative margins. Thus, they concluded that NIR with ICG can play a role in laparoscopic pancreatic resection of pNETs. Table 1 summarizes the results of the aforementioned studies.

\section{Discussion}

Pancreatic neuroendocrine tumours are rare tumours with indolent growth and a propensity to metastasize. There is vast heterogeneity on how they appear; some of them present with debilitating hormonal syndromes and bulky liver metastatic burden, whereas others may be asymptomatic, incidentally discovered via imaging studies [6]. Surgery remains the cornerstone of treatment, and as far as patients with localized disease are concerned, resection may be curative. Even patients with large metastatic liver deposits seem to benefit, in terms of symptom control and survival, after debulking surgical operations [27].

Computed tomography and MRI have been employed with varying success rates depending on the tumour subtype. Although these imaging modalities have a very high rate of detection for larger tumours, the results for smaller tumours are not optimal [28]. Visual inspection, manual palpation, and intraoperative ultrasound are utilized nowadays in order to identify pancreatic lesions and their borders intraoperatively. Nonetheless, a significant proportion of pNETs are not identifiable with the conventional imaging techniques. Failure to localize PNETs occurs in up to $30 \%$ of patients with gastrinoma and 10\% with insulinoma [5].

In this direction, ICG fluorescence imaging may facilitate the efforts of surgeons to identify occult pancreatic neuroendocrine lesions [19]. Invisible NIR fluorescent light, capable of penetrating millimetres into living tissue, is advantageous because it offers no change to the naked eye appearance of the surgical field, high-resolution images, and no significant increase in time of surgical operation. Newer imaging systems have been developed such as the FLARE ${ }^{\mathrm{TM}}$ system, which is also capable of displaying surgical anatomy with 2 independent channels of NIR fluorescence simultaneously [29].

This imaging modality might further help in the identification of resection margins and in cases of R1 resections, it could guide surgeons to operate further, as reported by Paiella et al. [22]. Newton et al. [17] concluded that NIR with ICG may provide meaningful tumour demarcation that correlates with tumour margins. Moreover, they reported that this technology may also be a promising way to assess the response to neoadjuvant treatment. Several studies also reviewed the use of NIR with ICG to assess its role within the sentinel node mapping; such studies are required in order to justify its use in these cases. Furthermore, the common bile duct and biliary anastomoses can easily be visualized during ICG-fluorescence study in cases of pancreatic resections [30]. Therefore, these imaging strategies could also be beneficial during PS in cases where the surgical anatomy is difficult to identify [16]. 
Yokoyama et al. [31] reported successful intraoperative detection of liver metastatic lesions (of at least $1.5 \mathrm{~mm}$ of diameter) in up to $16 \%$ of patients undergoing pancreatic resection without preoperative identifications of metastatic disease. Also, several preclinical studies have proven the efficacy of ICG fluorescence in the identification of pancreatic tumours in animal models $[32,33]$. Based on the available scientific data, pNETs were visualized because they are hypervascular lesions when compared to the surrounding parenchyma [19, 22]. Thus, the correct timing of ICG administration seems to be intraoperative, because there are no influx and efflux transporters in the pancreatic tissues and lesions [34].

\section{Conclusions}

Indocyanine green fluorescence might play a pivotal role in PS for PNETs. However, further large-scale clinical studies are needed to assess the optimal dosage time of administration of ICG in order to maximize the positive results. The broad utilization of this technique, by increasing negative margin specimens and by leading surgeons to proceed to adequate lymphadenectomy during pancreatic resections, might affect long-term survival of these patients.

\section{Disclosure}

The authors report no conflict of interest.

\section{References}

1. Schimmack S, Svejda B, Lawrence B, Kidd M, Modlin IM. The diversity and commonalities of gastroenteropancreatic neuroendocrine tumors. Langenbecks Arch Surg 2011; 396: 273-298.

2. Da Silva Xavier G. The cells of the islets of langerhans. J Clin Med 2018; 7: 54.

3. Fottner C, Ferrata M, Weber MM. Hormone secreting gastro-entero-pancreatic neuroendocrine neoplasias (GEP-NEN): when to consider, how to diagnose? Rev Endocr Metab Disord 2017; 18: 393-410.

4. Kuo JH, Lee JA, Chabot JA. Nonfunctional pancreatic neuroendocrine tumors. Surg Clin North Am 2014; 94: 689-708.

5. Winer JH, Choi HS, Gibbs-Strauss SL, Ashitate Y, Colson YL, Frangioni JV. Intraoperative localization of insulinoma and normal pancreas using invisible near-infrared fluorescent light. Ann Surg Oncol 2010; 17: 10941100.

6. Scott AT, Howe JR. Evaluation and management of neuroendocrine tumors of the pancreas. Surg Clin North Am 2019; 99: 793-814.

7. Sundin A, Arnold R, Baudin E, et al. ENETS consensus guidelines for the standards of care in neuroendocrine tumors: radiological, nuclear medicine \& hybrid imaging. Neuroendocrinology 2017; 105: 212-244.

8. Dromain C, de Baere T, Lumbroso J, et al. Detection of liver metastases from endocrine tumors: a prospective comparison of somatostatin receptor scintigraphy, computed tomography, and magnetic resonance imaging. J Clin Oncol 2005; 23: 70-78.

9. Puli SR, Kalva N, Bechtold ML, et al. Diagnostic accuracy of endoscopic ultrasound in pancreatic neuroendocrine tumors: a systematic review and meta analysis. World J Gastroenterol 2013; 19: 3678-3684.

10. Reubi JC. Somatostatin and other Peptide receptors as tools for tumor diagnosis and treatment. Neuroendocrinology 2004; 80 Suppl 1: 51-56.

11. McAuley G, Delaney H, Colville J, et al. Multimodality preoperative imaging of pancreatic insulinomas. Clin Radiol 2005; 60: 1039-1050.
12. Vahrmeijer AL, Hutteman M, van der Vorst JR, van de Velde CJH, Frangioni JV. Image-guided cancer surgery using near-infrared fluorescence. Nat Rev Clin Oncol 2013; 10: 507-518.

13. Keereweer S, Kerrebijn JDF, van Driel PBAA,et al. Optical image-guided surgery - where do we stand? Mol Imaging Biol 2011; 13: 199-207.

14. Frangioni JV. New technologies for human cancer imaging. J Clin Oncol 2008; 26: 4012-4021.

15. Jiang T, Olson ES, Nguyen QT, et al., Tumor imaging by means of proteolytic activation of cell-penetrating peptides. Proc Natl Acad Sci U S A 2004; 101: 17867-17872.

16. Hutteman M, van der Vorst JR, Mieog JSD, et al. Near-infrared fluorescence imaging in patients undergoing pancreaticoduodenectomy. Eur Surg Res 2011; 47: 90-97.

17. Newton AD, Predina JD, Shin MH, et al. Intraoperative near-infrared imaging can identify neoplasms and aid in real-time margin assessment during pancreatic resection. Ann Surg 2019; 270: 12-20.

18. Maeda H, Maeda H, Wu J, Sawa T, Matsumura Y, Hori K. Tumor vascular permeability and the EPR effect in macromolecular therapeutics: a review. J Control Release 2000; 65: 271-284.

19. Shirata C, Kawaguchi Y, Kobayashi K, et al. Usefulness of indocyanine green-fluorescence imaging for real-time visualization of pancreas neuroendocrine tumor and cystic neoplasm. J Surg Oncol 2018; 118: 1012 1020.

20. Gotoh K, Yamada T, Ishikawa O, et al. A novel image-guided surgery of hepatocellular carcinoma by indocyanine green fluorescence imaging navigation. J Surg Oncol 2009; 100: 75-79.

21. Mitsuhashi N, Kimura F, Shimizu H, et al. Usefulness of intraoperative fluorescence imaging to evaluate local anatomy in hepatobiliary surgery. J Hepatobiliary Pancreat Surg 2008; 15: 508-514.

22. Paiella S, de Pastena $M$, Landoni L, et al. Is there a role for near-infrared technology in laparoscopic resection of pancreatic neuroendocrine tumors? Results of the COLPAN "colour-and-resect the pancreas" study. Surg Endosc 2017; 31: 4478-4484.

23. Terasawa M, Ishizawa T, Mise $Y$, et al. Applications of fusion-fluorescence imaging using indocyanine green in laparoscopic hepatectomy. Surg Endosc 2017; 31: 5111-5118.

24. Fengler J. Near-infrared fluorescence laparoscopy--technical description

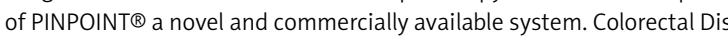
2015; 17 Suppl 3: 3-6.

25. Kawaguchi Y, Sugawara Y, Ishizawa T, et al. Identification of veno-occlusive regions in a right liver graft after reconstruction of vein segments 5 and 8: application of indocyanine green fluorescence imaging. Liver Transpl 2013; 19: 778-779.

26. Kawaguchi $Y$, Nomura $Y$, Nagai $M$, et al. Liver transection using indocyanine green fluorescence imaging and hepatic vein clamping. Br J Surg 2017; 104: 898-906.

27. Finkelstein P, Sharma R, Picado O, et al. Pancreatic neuroendocrine tumors (panNETs): analysis of overall survival of nonsurgical management versus surgical resection. J Gastrointest Surg 2017; 21: 855-866.

28. Abboud B, Boujaoude J. Occult sporadic insulinoma: localization and surgical strategy. World J Gastroenterol 2008; 14: 657-665.

29. Troyan SL, Kianzad V, Gibbs-Strauss SL, et al. The FLARE intraoperative near-infrared fluorescence imaging system: a first-in-human clinical trial in breast cancer sentinel lymph node mapping. Ann Surg Oncol 2009; 16: 2943-2952.

30. Boni L, David G, Mangano A, et al., Clinical applications of indocyanine green (ICG) enhanced fluorescence in laparoscopic surgery. Surg Endosc 2015; 29: 2046-2055

31. Yokoyama N, Otani T, Hashidate $H$, et al. Real-time detection of hepatic micrometastases from pancreatic cancer by intraoperative fluorescence imaging: preliminary results of a prospective study. Cancer 2012; 118: 2813-2819.

32. Von Burstin J, Eser S, Seidler B, et al. Highly sensitive detection of earlystage pancreatic cancer by multimodal near-infrared molecular imaging in living mice. Int J Cancer 2008; 123: 2138-2147.

33. Tran Cao HS, Kaushal S, Lee C, et al. Fluorescence laparoscopy imaging of pancreatic tumor progression in an orthotopic mouse model. Surg Endosc 2011; 25: 48-54.

34. Kitao A, Zen Y, Matsui O, et al., Hepatocellular carcinoma: signal intensity at gadoxetic acid-enhanced MR Imaging--correlation with molecular transporters and histopathologic features. Radiology 2010; 256: 817-826. 